\title{
Elaboración y validación de un cuestionario (CFVALMA), sobre la formación en valores del alumnado de magisterio Questionnaire design and validation (CFVALMA), on the training in values of teacher education students

\author{
Jose Ignacio Soto González, Diego Collado Fernández, Beatriz Torres Campos, Rosario Padial Ruz \\ Universidad de Granada (España)
}

\begin{abstract}
Resumen. La preocupación por la formación en valores es un tema de actualidad y considerado muy importante dentro de laformación inicial de los futuros docentes. El objetivo principal de este estudio es la el aboración de un cuestionario mediante el uso de latécnica D elphi para indagar acerca del conocimiento del alumnado de la Facultad de Ciencias de la Educación y la Escuela U niver sitariade Magisterio LaInmaculada, pertenecientesalaU niversidad de Granada, sobre laformación en valores recibida. Se usó una metodología descriptivatransversal graciasalatécnicaD elphi $(n=9)$ para la el aboración del cuestionario. Se asegura la val idez del instrumento gracias a la revisión llevada a cabo, la colaboración del panel de expertosy el pilotaje del cuestionario. En cuanto a la fiabilidad, se obtiene un Alfa de Cronbach de .940, que garantiza la fiabilidad de la her ramienta, resultando una herramientaútil para el objetivo planteado.
\end{abstract}

Palabras clave: instrumentos de evaluación, cuestionarios, estudiantes, Educación superior, valores, formación docente, técnica Delphi.

\begin{abstract}
The terms of «values» it is a nowadays issue, and considered even more important within the tasks that university professors, in charge of their initial training, must carry out. The main objective of thisstudy is the elaboration of a questionnaire by using the Del phi technique to inquire about the knowledge of the teachers in training of the Faculty of Education Sciences and the University School of Teaching La Inmaculada, belonging to the U niversity of Granada, on the teaching in values received. A descriptive-transversal methodology for the elaboration of the questionnaire was used with Delphi technique ( $n$ $=9$ ). The validity of the instrument is ensured after the review carried out, the collaboration of the panel of experts and the pretest of the questionnaire. Regarding reliability, a Cronbach'sAl pha of . 940 was obtained, which guaranteesthe reliability of the tool, resulting in a useful tool for the stated objective.
\end{abstract}

Key words: assessment instruments, questionnaires, students, higher education, values, teacher training, Del phi technique.

\section{Introducción}

La educación en valores aparece como aspecto relevante en el funcionamiento de las sociedades desde la antigüedad, actualmente toma mayor importancia, si cabe, como labor prioritaria entre las funciones de la escuela. Las vertiginosas transformaciones tecnológicas, entre otra serie de factores, están influyendo en la vida de los individuos y, por ende, en el alumnado. En la actualidad, autores como Sebastiani (2019), reclaman métodos de enseñanza-aprendizaje que impliquen globalidad, significatividad, funcionalidad, autonomía e implicación cognitiva. Resulta innegable pensar en la futura ciudadanía y en la necesidad de una educación basada en el compromiso y en el poder de transforma ción personal y social. En el estudio de Rubio, Hernández

Fecha recepción: 22-08-20. Fecha de aceptación: 28-11-20 Jose Ignacio Soto González

sotogonzalezj@gmail.com
\& Varón (2019) se refieren al valor como la variable dependiente más importante en el estudio de la cultura y la sociedad, y la variable independiente principal en el estudio de las actitudes y comportamientos de la sociedad.

Tal es la importancia de este tema que la declara ción del Decenio de la Naciones U nidas sobre la Educa ción para el Desarrollo Sostenible (UNDESD), en el 2014, ofrece recomendaciones y directrices para incorporar principios de educación para el desarrollo sostenible dentro de las políticas y los programas educativos, haciendo referencia a los desafíos a los que se enfrenta el mundo, destacando la gestión ambiental, el aprendizaje social, las competencias, la ética y la organización de los estudiantes (Cebrián \& Junyent, 2015).

Latorre \& Blanco en 2009, destacan que, pese al crecimiento de la investigación sobre la formación inicial del profesorado, hay aspectos que han tenido menos relevancia, como puede ser la formación práctica universitaria de los futuros docentes, puntualizando aún más, 
el tipo de creencias que los aprendices de profesor tienen al respecto.

Actualmente se reconoce al profesorado como eje del sistema educativo recayendo sobre sus hombros la mayor parte de la responsabilidad de educar en valores, pero, nos cuestionamos al igual que Azkarate, Bartau \& Lizasoain (2019), ¿está el profesorado suficientemente formado?, ¿se contempla en el período formativo del profesorado la formación en educación en valores?, ¿se forma al profesorado en estrategias de transmisión de valores?

Detal manera, pretendemos con nuestraherramienta poder explorar las creencias del profesorado en ma teria de formación en valores y, de esta manera, estaremos más cerca de lo que para ellos significa la escuela como institución, el papel de la educación en la sociedad y su función como docentes. Esto supone dimensiones relacionadas entre las creencias de los profesores/ as y las repercusiones que las mismas pueden tener en sus prácticas de enseñanza (Beck, Czerniat \& Lumpe, 2000), ya que todo el profesorado educa en valores enriqueciendo el desarrollo integral del alumnado y dando respuesta a los desafíos de la sociedad (Azkarate et al., 2019; Cepero, García \& López, 2013; Zbar, Brown, Bereznicki \& Hooper, 2003). Pensamos que la acción docente constituye uno de los aspectos esenciales en los que se ha de incidir con el fin de obtener resultados exitosos en el proceso de enseñanza y aprendizaje (González \& López, 2010; M ergler \& Spooner-Lane, 2012).

\section{Enseñanza superior y valores}

Ya desde el documento de Política para el Cambio y el Desarrollo de la Educación Superior realizado por la UNESCO en 1995, nos señala la necesidad imperiosa de que los centros de educación superior desempeñen un papel aún mayor en el fomento de los valores éticos y morales en la sociedad, y dediquen especial atención a la promoción, entre los futuros graduados, de un espíritu cívico de participación activa, reclamada por la sociedad.

Por esta razón, la acción de la universidad no debe limitarse a la transmisión del saber; debe generar opinión, demostrar su compromiso con el progreso social y ser un ejemplo para su entorno (Soto, 2011).

Nos encontramos integrados en el Espacio Europeo de Educación Superior, el cual establece su énfasis en el aprendizaje de los estudiantes y el desarrollo de competencias, nos aproxima a un nuevo modelo educativo que pone el acento en la funcionalidad del aprendizaje, la autonomía y protagonismo de los estudiantes y en la labor facilitadoray gestoradel docente, abriendo la puerta a la participación y al establecimiento de nuevos compromisos desde los que hacer frente a la mejora de la formación práctica (Cepero, Marín \&Torres, 2010; López \& Hinojosa, 2008; Romero, Campoy, Machado \& Zagalaz, 2019).

La preocupación por el ámbito de la educación en valores en ciencias sociales la podemos situar en la segunda mitad del siglo XX, sin embargo, es en las décadas de 1980 y 1990 cuando se comienza a hablar de la investigación empírica sobre los valores en la literatura especial izada (Estebaranz, 1991). Encontramos estudios descriptivos real izados mediante la observación directa (Beem \& Brugman, 1985), o a la observación indirecta (Gervilla, 1987) y la medición de valores a través de escalas, cuestionarios, test (GarcíaH oz, 1976) o encuestas (Santamarta, 1985). U nos años más tarde, encontramos investigaciones desde un punto de vista antropológico, en el que se plantea una investigación de tipo etnográfico (Estebaranz, 1991). Todos estos estudios han servido de base para estudios como el nuestro. Sin embargo, encontramos que la mayoría de los cuestionarios van encaminados a disponer un sistema de constructos dignos de estima y los sujetos tienen que emitir un juicio de valor, mientras que en nuestro cuestionario pretendemos acercarnos a la opinión de los alumnos e indagar sobre cómo se les están transmitiendo estrategias y herramientas eficaces para ponerlas en práctica el día de mañana en su labor docente.

Entre las investigaciones más actuales fruto de la revisión de la revistas especializadas que utilizan el cuestionario para la evaluación de valores o actitudes, cabe destacar los estudios llevados a cabo por expertos en estas prácticas (por ejemplo, Benítez, 2009; Bosch, 2002; Gómez del Valle, 2003; González, 2003; González, Zurita, Pérez, Padial, U bago, \& Chacón, 2019; Hernández, Aciego \& Domínguez, 1994; León \& Crisol, 2011; Villaverde, González \& Toja, 2017), que aportan información sobre creación de herramientas en espacios de Educación Superior.

\section{La Formación en valores del alumnado de Ma- gisterio}

Existe una relación entre la educación en valores y la eficacia escolar, señalándose que este tipo de educación es muy efectiva en la construcción del carácter del alumnado y en la mejora de los resultados académicos (Azkarate, Bartau \& Lizasoain, 2019; Baños\& Arrayales, 2020; Barba, 2007). Es por esto que se reafirmalanece- 
sidad de formar al futuro profesorado en este ámbito. Autores como Ariza \& Q uevedo (2013), señalan que son menos de un $5 \%$ los trabajos de investigación en Ciencias de la Educación sobre el tema de la formación inicial del profesorado. Surge por tanto la necesidad de repensar el conjunto de conocimientos, actitudes, habilidades, valores y competencias que debe poseer un docente para iniciar su tarea profesional en buenas condiciones, siendo la formación en valores un aspecto a incluir en la formación inicial (Martínez, 2016).

Cámara (2009) establece un sistema de categorías paraestaformación, primero la Formación personal que desarrolle las características peculiares del alumnado, que le ayude a conocerse, valorarse, identificarse; le proporcione la madurez y el equilibrio afectivo y emocional necesarios para el desempeño de la tarea docente; le ayude a diseñar su escal a de valores, conocer otros y establecer diferencias entre ellos; la segunda la Formación intelectual, que comprende conocimientoscientíficos y culturales de las áreas curriculares y de enseñanza; conocimientos psicopedagógicos; conocimientos curriculares; fundamentosteóricos pedagógicosquejustifiquen su actuación en el aula; desarrollo de las capacidades personales, y por último laFormación social, como ser social, la relación con los demás, convivencia; rela ción con el contexto y el medio ambiente,... ; esta dimensión hace efectiva la función social de la educa ción en cuanto que transformadora y conservadora de los valores culturales, a la vez que capacita para vivir con los otros, desarrolla valores sociales y enseña a los alumnos/ as a trabajar en equipo, colaborar, tomar decisiones, resolver conflictos.

El comienzo del Espacio Europeo de Educación Superior (EEES) podemos señalarlo a partir de importantes citas como la Declaración de Bolonia (1999), la Declaración de Praga (2001) o el comunicado de Bergen (2005), en el que se tratan aspectos educativos en el ámbito de Europa (Coterón, Franco \& Gil, 2012). En España, surge a su vez la necesidad de adaptarse a la norma europea y comienzan los cambios en legislación. Magisterio adapta sus planes de estudio y los estudiantes obtienen un título generalista con un itinerario 0 mención, con un total de 30 créditos de carácter optativo.

En la actualidad en la U niversidad de Granada, tras la revisión de los planes de estudios, no contempla de forma específica ninguna formación para maestros/ as en el campo de laformación en val ores o de cómo transmitir una enseñanza en val ores al alumnado de prima ria(Soto, 2011).

\section{M etodología}

\section{Diseño y muestra}

Se trata de un estudio descriptivo transversal de medición única e instrumental (Almonacid-Fierro, Feu \&Vizuete, 2018; Ato, López \& Benavente, 2013), cuyo objetivo fue elaborar y validar un cuestionario (CFVALMA), adecuado para tener información de los conocimientos y creencias del al umnado de magisterio en relación con la formación en valores recibida en un centro público y otro privado de la U niversidad de Granada. Con este instrumento comprobamos el grado de conocimiento que el alumnado de magisterio de la provincia de Granada tiene sobre valores y actitudes éticas, y verificamos el conocimiento que dicho alumnado tiene de procedimientos y estrategias para educar en valores.

Este estudio se realizó en dos fases, primero la construcción del cuestionario y posteriormente su valida ción.

Para el pilotaje del estudio se eligió alumnado de segundo y tercero de magisterio de las diferentes especialidades: Educación Especial, Educación Física, Educa ción Primaria, Educación M usical, Educación Infantil y Lengua Extranjera, de la Facultad de Ciencias de laEducación, y de la Escuela Universitaria de Magisterio «La Inmaculada», ambos centros pertenecientes a la U niversidad de Granada.

El número de participantes fue de 417 alumnos/ as. La muestra se dividió en seis especialidades: 83 alumnos/ as de Educación Especial, lo que supone un 19,9\% de la muestra; 88 alumnos/ as de Educación Física, es decir 21,1\% del total; 171 alumnos/ as de Educación Primaria, que supone un 41\%; 23 alumnos/ as de Educación M usical, un 5,5\% de lamuestra; 31 de alumnos/ as deEducación Infantil, un 7,4\% del total; 21 alumnos/ as, un $5 \%$.

En la siguiente tabla, aparecen recogidos los datos de la muestra por centro de formación, por sexo y el rango de edad de los participantes.

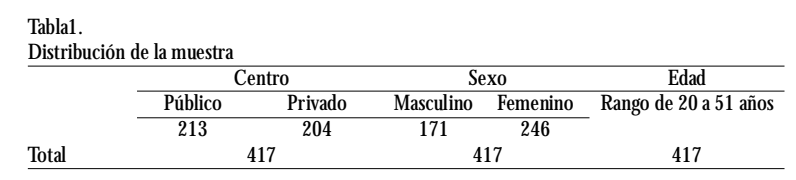

Según la determinación de la muestra de Arkin \& Colton (1984) teniendo en cuenta la población total, nuestra muestra cumple con el 95,5\% de margen de confianza, con un margen de error del $\pm 5 \%$. 


\section{Elaboración del instrumento}

Se decide la utilización del cuestionario como herramienta de recogida de información, la finalidad era explorar de manera sistemática y ordenada, las ideas y creencias del alumnado de magisterio de la Universidad de Granada. Podemos considerar que este tipo de instrumentos no son rechazados por el grupo a estudiar y se acerca a la realidad que queremos conocer (Rodríguez, Gil \& García, 1996).

Nuestro tipo de cuestionario también denominado inventario de creencias (Latorre \& Blanco, 2009), ha sido muy utilizado en numerosas investigaciones para recoger el pensamiento de estudiantes y futuros maestros/ as (Asensio \& Ruiz, 2017; Azkarate, Bartau, \& Lizasoain, 2019; Díaz-Barahona, Molina-García, \& M onfort-Pañego, 2019; Dunkin, Precians\& N ettle, 1994; Johnston, 1992; N ettle, 1998).

El cuestionario empleado en nuestra investigación consta de preguntas categorizadas, con lo que intenta mos hacer más exhaustivas las respuestas obtenidas (ta bla2). En nuestro caso, utilizamos este instrumento con formato escala likert, al igual que Chan \& Elliott (2004), para acercarnos a las creencias y pensamientos de los futuros maestros/ as sobre su formación en valores.

Para la elaboración y validación del cuestionario utilizamos la siguiente metodología:

1. Revisión bibliográfica

2. Técnica Delphi

3. Pilotaje del mismo

\section{Revisión bibliográfica}

El primer paso seguido para la elaboración del cuestionario fue una amplia y profunda revisión bibliográfica de lo investigado sobre creencias del estudiante y del profesorado en educación en valores. De esta manera pretendemos conseguir un profundo conocimiento del campo de investigación y crear las categorías para proponer a los expertos en la construcción del cuestiona rio, destacando las relacionadas con el objeto de estudio como Benítez (2009), Bosch (2002), Gómez del Valle (2003), González (2003), González, Zurita, Pérez, Padial, U bago, \& Chacón (2019), Hernández, Aciego \& Domínguez (1994), León \& Crisol (2011) yVillaverde, González \& Toja (2017).

Además, realizamos una revisión bibliográfica tanto de las publicaciones periódicas como de los textos existentes de los principales investigadores en el campo de la educación en valores a través de técnicas cuantitativas como puede ser el cuestionario o inventario de creencias, elaborados a partir de la bibliografía e investiga ciones, que aportan información sobre creación de herramientas en espacios de Educación Superior.

\section{Técnica Delphi}

Utilizamos estaTécnica como método de investigación sociológica perteneciente al tipo de entrevista de profundidad en grupo. Analizamos el estado de opinión sobre un tema, recabando la información de un grupo de individuos, expertos en el tema. Partiendo de las opiniones, puntos de acuerdo y discrepancias entre expertos, se puede llegar a ciertos elementos de consenso y una jerarquización de los aspectos importantes y secundarios del problema de estudio (Barrientos, 2001; Varela, 1991). Una de las ventajas de este método para nuestra investigación es que es un proceso sistemático, interactivo y grupal encaminado hacia la obtención de las opiniones y del consenso, a partir de las experiencias y juicios subjetivos de un grupo de expertos (Pozo, Gutiérrez \& Rodríguez, 2007; Scapolo \& Miles, 2006). $G$ racias a este método conseguimos extraer y maximizar las ventajas que presentan los métodos basados en grupos de expertos y minimizar sus inconvenientes (Martínez, 2003; Martínez, Soto \&W inter, 1982). Mediante esta técnica conseguimos obtener la opinión de consenso del grupo de una manera más fiable (ReguantÁlvarez \& Torrado-Fonseca, 2016).

\section{La selección del panel de expertos}

El instrumento fue validado mediante un procedimiento metodológicamente adecuado. Se usó un proceso de validación de contenido mediante juicio de expertos. Para la selección del panel de expertos, en primer lugar, se definió un universo de participantes posibles. La definición del universo está íntimamente relaciona da con el objetivo del proceso y con el tema en estudio (López, 2018). Posteriormente, se siguieron unos criterios de experticidad (criterio externo de evaluación), gra cias a los cuales se seleccionaron un grupo de nueve expertos, según el nivel de experiencia, reputación, disponibilidad, motivación e imparcialidad (Escobar \& Cuervo, 2008). Todos los expertos cumplían con al menos 4 de los 6 criterios establecidos:

- Doctores

- Profesores universitarios

- Profesores de educación secundaria o educación primaria.

\section{Asesores de formación}

Experiencia mínima de 10 años en docencia.

- Publicaciones en el área de conocimiento de educación en valores. 
Fases de la elaboración del cuestionario

Las fases de elaboración del cuestionario fueron las siguientes:

- Envío del primer cuestionario abierto.

- Análisis del primer cuestionario.

- Segundo cuestionario: jerarquizar la importancia de los temas y grado de acuerdo-desacuerdo con cada tema.

- Tercer cuestionario: argumentos y contra-argumentos.

Diseño y pilotaje del Cuestionario final.

\section{Envío del primer cuestionario}

Como ya hemos indicado anteriormente tras la revisión bibliográfica enviamos a los expertos un primer cuestionario abierto, en el cual se muestran los campos o dimensiones que hemos concluido que debía de tener este, con una serie de ítems acerca de las cuestiones más relevantes que nos interesaban evaluar en nuestra investigación. El procesamiento de las respuestas es tomado como base para la presentación de los siguientes cuestionarios. Realizamos tres vueltas y en la última recabamos la postura definitiva en función de los argumentos y contraargumentos expuestos por los distintos expertos. La redacción del primer cuestionario es abierta, facilitando que los expertos puedan responder con la máxima libertad de enfoque de jerarquización de la temática, de vocabulario y de terminología.

El primer cuestionario se remitió a través de correo electrónico, a un total de 10 expertos. Al principio del correo explicábamos brevemente los objetivos de nuestra investigación además de agradecer la colabora ción. De los 10 expertos con los que se contactó terminaron el proceso completo un total de 9 .

\section{Análisis de los resultados del primer cuestio-} nario

Una vez recibidas las respuestas del primer cuestionario caracterizadas por ser abiertas para que los expertos señalasen y anotasen todo lo que creyeran oportuno, realizamos su categorización y sistematización a través de una evaluación manual, ya que el volumen de información no era excesivo ni complicado para su procesamiento (Cea, 2001; González \& López, 2010; Palomares, 2003). Gracias a la información aportada por los expertos surgen las categorías del cuestionario.

La información recogida se puede sintetizar de la siguiente manera:

Preguntas de información acerca de los datos básicos de los alumnos para poder clasificarlos.
- Preguntas acerca del conocimiento sobre valores.

Preguntas sobre cómo les están transmitiendo sus profesores los valores.

- Preguntas sobre qué conocimiento tienen de herramientas, estrategias y recursos eficaces en la transmisión de valores.

\section{Análisis de los resultados del segundo cuestio- nario}

Recibidas las respuestas de los 10 expertos participantes en el segundo cuestionario, el análisis de las preguntas está estructurado en tres grupos de opinión:

- Puntos de consenso o acuerdo entre la mayoría de los expertos.

- Discrepancias.

- O tras opiniones sugeridas que no se contradicen con las de los demás.

En general las matizaciones son sobre los siguientes aspectos:

- Formular todos los ítems con un verbo en primera persona de manera que se realiza una afirmación la cual tendrá que valorar el encuestado.

- Eliminar aquellosítems parecidos o que no aportan ninguna información relevante.

- Corregir ítems de dudosa interpretación o que pueden llevar a confusión.

- Utilizar diferentes escalas de valoración de cada uno de los ítems.

- Usar un lenguaje más inclusivo, utilizando el femenino y el masculino en algunas palabras.

- Corregir la redacción y formulación de algunos ítems por errores gramaticales.

Todos los expertos menos dos están de acuerdo con las categorías enunciadas.

\section{Análisis del tercer cuestionario}

La tercera vuelta se inicia tres meses más tarde, remitiendo el cuestionario a cada uno de los panelistas por e-mail. En esta tercera vuelta, hemos contrastado las discrepancias identificadas en el segundo cuestiona rio. Se somete a la evaluación de los panelistas aquellas

Tabla 2

Campos del cuestionario definitivo.

Campo 1: Información general.

Año de nacimiento.

Sexo.

Curso que estudia

Especialidad que estudia.

Campo 2: Conocimiento sobre los val ores.

15 ítems.

Campo 3: Cómo les están transmitiendo sus profesores los valores

12 ítems.

Campo 4: Conocimiento sobre herramientas, estrategias y recursos eficaces en la transmisión de valores.

20 ítems. 
ideas que son de interés para el tema de estudio y que fueron planteadas por los panelistas, en especial priorizando sobre los aspectos fundamentales de cada dimensión. Los resultados más importantes son:

\section{Resultados}

\section{Validez del Cuestionario}

A seguramos la val idez interna del cuestionario apoyándonos en una amplia y profunda revisión teórica y documental de los cuestionarios que se han utilizado previamente con un objeto de estudio similar al nuestro. Garantizamos la validez externa apoyándonos en dos procedimientos: lavalidez de los expertosy laprueba piloto del cuestionario (Bell, 2002; Latiesa, 1996; Losada \& López-Feal, 2003).

Los expertosquehan participado en la técnicaD elphi han interpretado el significado de los ítems y han valorado su claridad, pertinencia y coherencia. Cada uno de los expertos tiene su propio estilo, definición y perspectiva del objeto de estudio, y esto se refleja en los datos resultantes, lo que nos puede llevar a datos más válidos.

La prueba piloto, además de mirar los primeros índices de fiabilidad de los ítems y consistencia del cuestionario, también aportó indicadores sobre el grado de comprensión y de acuerdo de los participantes en esta fase previa con los significados e ítems que se proponían.

Según Cea (2001), para la prueba piloto del cuestionario, se escoge una pequeña muestra de individuos de iguales características que la población estudio. En nuestro caso la prueba piloto la realizamos con un total de 60 individuos $(n=60)$, al umnos de segundo y tercer curso de la Escuela U niversitaria La Inmaculada. El objetivo esencial de esta fórmula de validación es evaluar la adecuación del cuestionario, la formulación de las preguntasy su disposición conjunta. En concreto, con estaprueba pretendimos comprobar que:

- Las preguntas tienen sentido, se comprenden y provocan las respuestas esperadas.

- La disposición conjunta del cuestionario (su secuencialógica) es adecuada y que su duración no fatigue al cuestionado.

- Las instrucciones que figuran en el cuestionario se entienden.

\section{Fiabilidad del Cuestionario}

La fiabilidad de nuestro cuestionario se calculó mediante el coeficiente de Alpha de Cronbach del Cues- tionario Piloto, pasado a un grupo de 60 individuos(Bell, 2002; Del Villar, 1994; M cM illan \& Schumacher, 2005). Para ello utilizamos el paquete estadístico S.P.S.S. 19.0 (Statistical Package for the Social Sciences). Este programa nos facilitó los coeficientes, obteniendo un Alfa de Cronbach en todos los ítems por encima de . 6 , indicándonos que el cuestionario es fiable. Los resultados del análisis de fiabilidad global de los ítems del pilotaje, del uno al 47 arrojan un valor de fiabilidad de .940, refiriéndonos a la consistencia interna del instrumento.

El resultado pormenorizado del Alfa de Cronbach del Cuestionario Piloto, se expone en la tabla 4:

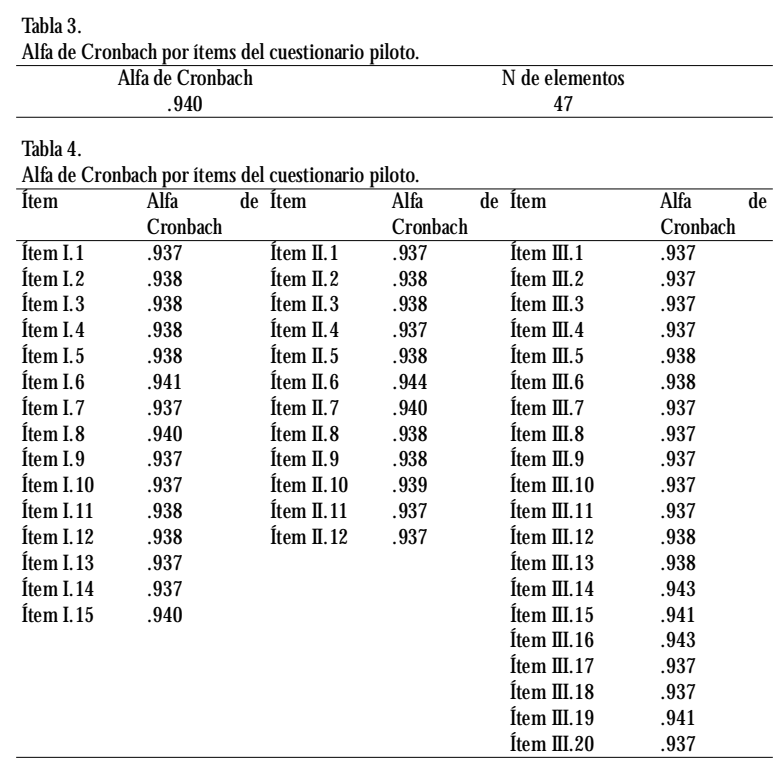

\section{Discusión}

Partiendo de los objetivos generales que formaban parte de un proceso de investigación más amplio, en esta publicación se muestra el trabajo llevado a cabo en la elaboración y validación de un cuestionario adecuado para acercarnos a los conocimientos y creencias del alumnado de magisterio en relación a la formación en valores recibida tanto en un centro público y otro privado de la U niversidad de Granada.

Para la elaboración del cuestionario se utilizó la técnica Delphi, ya que como señalan Linstone \& Turoff (1975), el Delphi puede ser caracterizado como un método para estructurar el proceso de comunicación grupal, de modo que ésta sea efectiva para permitir a un grupo de individuos, como un todo, tratar con problemas complejos. Helmer (1983), define el método como un programa cuidadosamente elaborado, que sigue una secuencia de interrogaciones individuales a tra vés de cuestionarios, de los cuales se obtiene informa ción que constituirá la retroalimentación para los cues- 


\section{tionarios.}

Algunos condicionantes esenciales tenidos en cuenta para el éxito del grupo Delphi fueron que se dispuso de un tiempo relativamente largo (tres meses). Además, el contacto con los participantes fue por escrito y demostraron todos ellos un interés continuado, ya que el proceso requiere de una alta implicación de los participantes. Posteriormente, el personal investigador encargado de la encuesta deberá dar prueba de la competencia en la interpretación de las respuestas obtenidas y en la toma de decisiones. La principal desventaja que encontramos en el método, estuvo relacionada con la información sobreel propio procedimiento, ya que la técnica en sí no especifica si el anonimato entre los participantes debe de ser respetado entre el grupo de análisis. Tampoco se especifica cuál debe de ser el mínimo de participantes cuando el grupo es heterogéneo (Paloma res, 2003).

Entre las ventajas que valoramos de este tipo de metodología señalamos que es una técnica que dejatiempo para la reflexión; el anonimato evita las influencias entre los miembros del panel; no se concede ningún privilegio a los intereses particulares, puesto que se devuelve de forma sistemática toda información a los participantes; el participante es libre de responder en el momento que le conviene y no tiene que desplazarse; se logra consenso sobre puntos de vista dispares sobre unos mismos elementos; la complementariedad con otras técnicas. La técnica Delphi tiene especial utilidad cuando se trabaja con elementos al tamente subjetivos sobre los que resulta difícil determinar su valor intrínseco. La técnica, facilita lograr consenso entre puntos de vista muy dispares sobre unos mismos elementos. No obstante, el Delphi es susceptible de modificaciones cuando se utiliza en determinados estudios de investigación. Cabe, pues, la posibilidad de entregar a los panelistas de la primera circulación un cuestionario ela borado a partir de los trabajos de otros expertos y sustentados en otras técnicas de investigación cualitativas como brainstorming, grupo nominal, grupo de discusión, grupo focal...

Entre las limitaciones de fondo que observamos nos gustaría destacar: los prejuicios del monitor, ideas preconcebidas de parte del grupo monitor conducirán a la elección de un panel que tienda a asegurar resultados en la dirección. También puede llevar a plantear preguntas dirigidas o restringidas, que no agotan todas las posibilidades de investigación del tema en estudio. M anipula ción de datos: este método por el carácter anónimo de las respuestas y por la forma en que se procesan está dado a esta manipulación. Ignorar desacuerdos: una de las características de este método es la exploración de los desacuerdos. Estos pueden ser ignorados cuando el criterio de lo que se considera consenso es muy flexible, ello conduciráa resultados sesgados y de escasa contabilidad; Sobreestimación de la técnica: autores como Linstone \& Turoff (1975) afirman que una proliferación de las aplicaciones incorrectas del método pueden tener consecuencias negativas y llevar al uso indiscriminado, con la consecuente repetición de estudios sobre un mismo tema, lo que signifique que cada nuevo estudio aporte muy poco conocimiento adicional sobre un tema, es lo que se ha denominado rendimiento decreciente.

\section{Conclusiones}

Se ha asegurado la validez de nuestro instrumento, tanto de manera externa, realizando una profunda revisión teórica y documental, como de manera interna con la validez de los expertos y la prueba piloto del cuestionario.

Garantizamos la fiabilidad de nuestro instrumento mediante el coeficiente de Alpha de Cronbach, realiza do en los 47 ítems del cuestionario piloto, obteniéndose un valor de .940 en cuanto a consistencia interna del instrumento, se refiere.

Señalar que la técnica Delphi ha dado grandes resultados en el campo de la investigación y muestra una alta fiabilidad y validez como ha quedado demostrado.

Así, podemos afirmar que nuestro cuestionario sobre conocimientos y estrategias de transmisión de va lores en alumnos/ as de magisterio está bien construido, siendo un instrumento útil y adecuado.

Nos gustaría concluir haciéndonos eco de las pala bras de De la O rden (1997) cuando indica la importancia de la labor del profesor/ a y del maestro/ a como miembro de una comunidad académica, lo que supone la aceptación y conformación de la conducta a un conjunto específico de pautas, valores y actitudes que, de alguna manera, reflejan una determinada percepción de la realidad y caracterizan y dan sentido a una forma de vida.

En la sociedad del conocimiento las universidades deben asumir su responsabilidad, transfiriendo aprendizajes a los futuros maestros/ as y estos a los educandos, para transformar la sociedad, a partir de una práctica educativa que otorgue una formación de excelencia (González, et al., 2020), y, en especial, en las carreras de formación inicial del profesorado. 


\section{Referencias}

Almonacid-Fierro,A., Feu, S. \&Vizuete, M. (2018).Validación de un cuestionario para medir el Conocimiento Didáctico del Contenido en el profesorado deEducación Física. Retos, nuevas tendencias en educación física, deporte y recreación 34, 132-137.

Ariza,T. \& Q uevedo, R. (2013).Análisis bibliométrico delarevista de investigación educativa (2000-2012). Revista de investigación educativa, 31(1), 31-52. https:/ / doi.org/ 10.6018/ rie. 31.1 .160321

Arkin, H. \& Colton, R. (1984). An outline of statistical methods as applied to economics, business, education, social and physical science. NewYork: Barnes \& Noble.

Asensio M uñoz, I. \& RuizdeM iguel, C. (2017). Mediday evaluz ción de las creencias sobre la profesión de los maestros en formación. Revista Electrónica Interuniversitaria de Formación del Profesorado, 20 (3), 79-91. https:/ / doi. org/ 10.6018/ reifop. 20.3.265231

Ato, M., López, J. J., \& Benavente, A. (2013). Unsistemade clasificación de los diseños de investigación en psicología. Anales de Psicología, 29(3), 1038-1059. https:/ / dx. doi. org/ 10.6018/ analesps. 29.3.178511

Azkarate, A., Bartau, I. \& Lizasoain, L. (2019). Educación en valores y formación del profesorado de secundaria: el caso de la UPV/ EHU. Profesorado: revista de curriculum y formación del profesorado, 23(3), 493-516.

Baños, R., \&Arrayales, E. (2020). Predicción del aburrimiento en la educación físicaa partir del climamotivacional. Retos. nuevas tendencias en educación física, deporte y recreación, 38, 83-88. https:/ / doi.org/ 10.47197/ retos. v38i38.74301

Barba, B. (2007).Valores, formación moral y eficacia escolar. Una revisión de la investigación educativa en M éxico. REICE: Revis ta Electrónica Iberoamericana sobre Calidad, Eficacia y Cambio en Educación, 5(5), 32-36.

Barrientos, J. (2001). Técnicas y Métodos de Investigación Social: M étodo Delphi, Aplicación en el campo sanitario. Departamento de CienciasPolíticas. Universidad de Granada. Trabajo propio del Departamento.

Beck, J., Czerniat, Ch. \& Lumpe, A. (2000). An exploratory study of teacher's beliefs regarding the implementation of constructivism in their classroom. Journal of Sciencie Teacher education, 11(4), 323-343.

Beem, A. L., \& Brugman, D. (1986). The Effects of Values Development Lessons on Pupils' Well-Being, Pleasure in School, Mutual Relationships, and on Pupils' Valuational Behavior during Classroom Dialogues. Theory \& Research in Social Education, 14(2), 97-112.

Bell, J. (2002). Cómo hacer tu primer trabajo de investigación: guía para investigadores en educación y ciencias sociales. Barcelona: Gedisa.

Benítez, A. (2009). La educación en valores en el ámbito de la educación superior. Revista Iberoamericana sobre Calidad, Eficacia y Cambio en Educación, 7(2), 116-129.

Bosch, M. C. (2002). Valores y creencias del profesorado de secundaria sobre algunosaspectos de la educación». Revisa Electrónica Interuniversitaria de Formación del Profesorado, 5(2).

Buendía, L. (1992). Técnicase instrumentos de recogidade datos.
En M. P. Colas y L. Buendía (Eds), Investigación Educativa. Sevilla: Alfar.

Cámara, A. M. (2009). Construcción de un instrumento de categorías para analizar valores en documentos escritos. REIFOP 12(2), 59-72.

Cea, M. A. (2001). Metodología cuantitativa: estrategias y ténicas de investigación social. Madrid: Síntesis.

Cebrián, G. \& Junyent, M. (2015). Competencies in Eduction for Sustainable Development: ExploringtheStudentTeachers' Views. Sustainability 7, 2768-2786; doi:10.3390/ su7032768.

Cepero, M., García, A. \& López, M. (2013). Diseño deun progra ma de intervención bilingüe para el área de Educación Física basado en la competencia digital. Porta Linguarum, 19, 257273.

Cepero, M., Marín, M. N. \&Torres, J. (2010). Teachingand learning social values: experience of resolution of conflicts in the classroom of physical education across the learning of social skills. Journal of human sport \& exercise, 5(3), 497-506.

https:// doi.org/ 10.4100/ jhse.2010.53.20

Chan, K. W. \& Elliott, R. G. (2004). Relational analysis of personal epistemology and conceptions about teaching and learning Teaching and TeacherEducation, 20, 817-831.

Coteron, J., Franco, E. y Gil, J. (2012). O pinión del alumnado sobre la implantación del Espacio Europeo de Educación Superior en estudios de Ciencias de laActividad Física y del Deporte. Revista Complutense de Educación, 23 (1), 191-206.

DeLaO rden,A. (1997). Desarrollo y validación deun modelo de calidad universitariacomo baseparasu evaluación. Revista Electrónica de Investigación y Evaluación Educativa, 3.

DelVillar, F. (1994). La credibilidad de la investigación cualitativa en la enseñanza de la educación física. Apunts. Educación Física y Deportes, 37, 26-33.

Díaz-Barahona,J., M olina-García,J. \& M onfort-Pañego, M. (2019). Estudio de las actitudesy el interés delos docentes de primaria de educación física por lasTIC en la ComunidadValenciana. Retos. nuevastendenciasen educación física, deportey recreación, 35, 267-272. https:/ / doi.org/ 10.47197/ retos.v0i35.63355

Dunkin, M. J., Precians, R. P. \& Nettle, E. B. (1994). Effects of formal teacher education upon student teachers' cognitions regarding teaching Teaching andTeacher Education, 10, 395-408.

Escobar, J., \& Cuervo, Á. (2008).Validez de contenido y juicio de expertos: una aproximación a su utilización. Avances en medidión, 6(1), 27-36.

Estebaranz, A. (1991). El cuestionario como instrumento de recogida de datos cualitativosen estudiosetnográficos. Un estudio sobre valores. Enseñanza, 8, 165-185.

García Hoz,V. (1976). El vocabulario general de orientación científica y sus estratos. (bases para una orientación vertebrada). Madrid. CSIC.

Gervilla, E. (1987). Losfines de la educación hoy. Reflexiones en torno a los planes de centro de Granada. Revista de ciencias de la educación (Madrid), (131), 333-339.

Gómez delValle, M. (2003). Identificación de los estilos de aprendizajepredominantesen estudiantes demagisterio de laFacultad de Ciencias de la Educación de la Universidad de Cádiz. Revida Electrónica Interuniversitaria de Formación del Profesorado, 
$6(2)$.

González, G. , Zurita, F., Pérez,A.J., Padial, R., U bago,J. L. \& Chacón, R. (2019). Estudio descriptivo del clima motivacional percibido haciael deporte según el sexo de losfuturos docentes de Educación Física. Sportis Sci J, 5(1), 85-100. https: / / doi.org/ 10.17979/ sportis. 2019.5.1.3479

González, I. \& López, A. B. (2010). Sentando las bases para la construcción de un modelo de evaluación a las competencias docentes del profesorado universitario. Revista de Investigación Educativa, 28 2), 403-423.

González, M., M olina, E., Beltrán, A., Flández, J., Negrón, M., Martínez, C., Vargas, R., Trujillo, H., Ferreira, M. . Leiva, R. \& Lobos, M. (2020). Construcción y validación de un instrumento paravalorar lacalidad de laformación de profesoresde Educación Física en Chile. Retos. nuevas tendencias en educación física, deporte y recreación, 37, 312-319. https:/ / doi.org/ 10.47197/ retos. v37i37.69304

González,V. (2003). Educar valores en la universidad: reflexiones desde una perspectiva psicológica. Revida cubana de psicología, 20(1), 50-58.

Helmer, O. (1983). Looking Forward:A Guideto FutureR escarch. Beverly Hills, CA: Sage.

Hernández, P., Aciego, R. \& Domínguez, R. (1994). Crecimiento personal y educación en valores sociales: evaluación del programa PIECAP. Revida de Psicología General y Aplicada, 47(3), 339-347.

Johnston, S. (1992). Images: a way of understanding the practical knowledge of student teachers. Teaching and Teacher Education, 8, 123-136.

Latiesa, M. (2000). Validez y fiabilidad de las observaciones sociológicas. El análisis de la realidad social. M étodos y ténicas de investigación, 409-443.

Latorre, M. J. \& Blanco, F. J. (2009). Lainvestigación sobrecreencias docentes através del método de encuesta. XXI. Revista de Educación, 11.

León, M.J. \& Crisol, E. (2011). Diseño de cuestionarios (OPPUMAUGR y OPEUMAUGR): La opinión y la percepción del profesorado y de los estudiantes sobre el uso de lasmetodologías activas en launiversidad. Profesorado. Revista de curriculum y formación del profesorado, 15(2), 271-298.

Linstone, A. \& Turoff, M. (1975). The Delphi Method:Technique and Applications. Massachusetts, MA: Addison-Wesley.

López, E. (2018). El método Delphi en la investigación actual en educación: unarevisión teóricaymetodológica. Educación XX1, 21(1), 17-40, doi: 10.5944/ educXX1.15536

López, M. C. \& Hinojosa, E. (2008). Percepciones iniciales de los estudiantes sobre la formación práctica. Revista Iberoamericana de Educación, 5(47).

Losada, R. \& López-Feal, R. (2003). Métodos de investigación en ciencias humanas y sociales Madrid:Thomson, D.L.

Martínez, E. (2003). LatécnicaD elphi como estrategiade consulta a los implicados en la evaluación de programas. Revista de Investigación Educativa 21(2), 449-463.

Martínez, C., Soto, E. \&W inter, J. (1982). M anual Delphi, Tesispara la obtención del título de Ingeniero Comercial. Departamento de Economía, Facultad de Ciencias Económicas y Administrati- vas de la Universidad de Chile.

Martínez, M. (2016). La formación inicial de los maestros: una responsabilidad compartida. Bordón 68(2), 9-16. https:/ / doi.org/ 10.13042/ Bordon.2016.68201

Mc Millan, J.H. \& Schumacher, S. (2005). Investigación educativa: una introducción conceptual. Madrid: Pearson Addison Wesley.

Mergler, A. G., \& Spooner-Lane, R. (2012). What Preservice Teachers need to know to be Effective at Values-based Education. Australian Journal of Teacher Education, 37(8).

Nettle, E. B. (1998). Stability and change in the beliefs of student teachers during practiceteaching Teaching andTeacher Education, $14,193-204$.

Palomares, J. (2003). Motivaciones, hábitos físco-deportivos y usos de los espacios del Parque Periurbano Dehesas del Generalife. Granada: Reprodigital.

Pozo, M.T., Gutiérrez, J. \& Rodríguez, C. (2007). El uso del método Delphi en la definición de los criterios para una formación de calidad en animación sociocultural y tiempo libre. Revista de Investigación Educativa 25(2), 351-366.

Reguant-Álvarez, M. \& Torrado-Fonseca, M. (2016). El método Delphi. REIRE, Revista d'Innovació i Recerca en Educació, 9(1), 87-102.

Rodríguez, G., Gil, J. \& García, E. (1996). M etodología dela investigación cualitativa. Málaga: Aljibe.

Romero, J. R., Campoy,T. J., M achado, F. \&Zagalaz, M. L. (2019). El docente universitario y la diversidad cultural en el Espacio Europeo de Educación Superior (EEES). Interdisciplinary Scientific Journal, 6(1), 177-192.

Rubio, G. A., Hernández, L. \&Varón, N. (2019).Validación de un cuestionario paraevaluar valores personalesy socialesen jóvenes deportistas. Retos. Nuevas tendencias en educación física, de portey recreación, (36), 152-158. https: / / doi.org/ 10.47197/ retos. v36i36.65253

SANTAMARTA, J. \& otros (1985): Los valores en el mundo de los jóvenes Juventud hoy, conflictos y esperanzas, Materiales y documentación, no. 10. Madrid, Consejo de Cultura, M. Huerta, pp. 125-153.

Sebastiani, E. M. (2019). Towards Committed Physical Education. Apunts. Educación Física y Deportes, 137, 3-4.

Scapolo, F. \& Miles, I. (2006). Eliciting experts' knowledge: A comparison of two methods. Technological Forecasting and Social Change, 73.

Soto, J. I. (2011). Conocimientos y creencias sobre la formación en valores y técnicas de intervención del alumnado de magisterio de Granada. Tesis Doctoral. Universidad de Granada.

UNESCO. World Conference on Education for Sustainable Development. Available online: http:/ / www.unesco.org/ new/ en/ unesco-world-conference-on-esd-2014

Varela, J. (1991). Los métodos de consenso en el sector sanitario. Gaceta sanitaria, 5(24), 114-116.

Villaverde, E. J., González, M. A., \&Toja, M. B. (2017). El buen profesor de Educación Física desde la concepción del alumnado universitario en Galicia. Sportis Sci I, 3(2), 286-302.

Zbar, V., Brown, D., Bereznicki, B., \& Hooper, C. (2003). Values education study: Final report. Melbourne: Curriculum corporation. 
Año de nacimiento:

Sexo: Masculino Femenino

Curso que estudias: Segundo Tercero

Centro donde estudias: FCCE Granada: $\quad$ EUMI: $\quad 0$ tro:

Especialidad:

CUESTIONARIO

Las casillas de la derecha coinciden con las siguientes valoraciones:Totalmente en desacuerdo (1), Algo de acuerdo (2), Medianamente de acuerdo (3), Bastante de acuerdo (4), Muy de acuerdo (5) Totalmente de acuerdo (6)

\section{CAM PO 1: Conocimiento sobre los valores}

I.1 Conozco el significado de valor en educación.

I.2 Tengo clara la diferencia entre valores y actitudes

1.3 Se diferenciar entre valor y norma social.

I.4 Se diferenciar entre valores y sentimientos.

1.5 Podría realizar una lista con un amplio número de valores.

I.6 Conozco las principal es teorías axiológicas

1.7 Tengo claras las características que tiene un valor.

1.8 Se la naturaleza de los valores.

1.9 Se formular objetivos referidos a valores

I.10 Se formular competencias teniendo en cuenta valores.

I.11 Soy consciente de la importancia de los val ores en nuestra cultura.

I.12 Puedo realizar una clasificación de valores.

1.13 Se diferenciar entre valores sociales y valores individuales

I.14 Considero que los val ores morales son exigibles en las relaciones de una per sona con otra.

I.15 Se diferenciar entre valores instrumental es y val ores ter minales.

CAM PO 2: Como les están transmitiendo sus profesores los valores

II.1 Considero que mi formación en valores por mis maestros/ as en primaria ha sido adecuaday satisfactoria

II.2 Considero que mi formación en valores por mis maestros/ as en secundaria ha sido adecuada y satisfactoria

II.3 Siempre he recibido además de una for mación en conceptos y procedimientos, una adecuada carga actitudinal.

II.4 Creo que la for mación en valores recibida en la carrera de magisterio es adecuada y satisfactoria.

II.5 Me gustaría recibir una formación tanto en conceptos, procedimientos como en actitudes.

II.6 M is profesores/ as le dan mucha más importancia a los contenidos conceptuales y procedimentales y muy poca a los actitudinales

11.7 Mis profesores/ as me han evaluado en actitudes y val ores.

II.8 Mis profesores/as de magisterio me transmiten un amplio bagaje en valores.

II.9 En el trato con mis profesores de magisterio reconozco valores que a mí me gustaría transmitir a mis futuros al umnos

II.10 El sistema educativo universitario favorece la transmisión de val ores entre profesor/ a y alumno/ a

II.11 En la titulación de magisterio se le da importancia a los valores.

II.12 Mantienen mis profesores/ as actitudes coherentes con las que quieren transmitir.

CAM PO 3: Conocimiento sobre herramientas, estrategias y recursos eficaces en la transmisión de valores

III.1 Tú, como futuro/ a maestro/ a de educación primaria crees que dominas estrategias de intervención en valores (para enseñar valores).

III.2 En los trabajos a realizar para las asignaturas de la car rera, he tenido en cuenta los valores que me qustaría transmitir a mis futuros alumnos/ as.

III. 3 Conozco mecanismos de transmisión y fijación de actitudes éticas en el desarrollo de la personal idad del alumnado.

III.4 Me gustaría recibir más formación para poder transmitir val ores como futuro maestro.

III.5 Creo que una mejor for mación en herramientas para transmitir valores a futuros docentes mejoraría la educación y los problemas disciplinares (convivencia). III. 6 Me planteo en qué medida estoy for mado/ a como maestro/ a para transmitir val ores.

III.7 Se utilizar los recursos que he aprendido para transmitir valores.

III. 8 Me han enseñado herramientas para transmitir valores.

III. 9 Me han enseñado recursos, instrumentos y herramientas para eval uar val ores.

III. 10 Considero que se podrían mejorar o cambiar problemas sociales con una adecuada for mación en valores.

III. 11 Considero los valores algo importante/ esencial en la formación del maestro/ a

III. 12 Creo que domino bastantes herramientas para transmitir val ores a mis futuros al umnos/ as

III.13 Considero la formación en valores como algo importante para mi futuro como maestro/ a.

III.14 Considero inadecuada la carga lectiva de educación en valores en los planes de estudio de maestros.

III.15 Se le da poca importancia a la educación en valores en los planes de for mación de futuros maestros.

III.16 En pocas asignaturas de la car rera se trabaja el aprendizaje y transmisión de valores

III. 17 Conozco técnicas para trabajar val ores.

III. 18 Conozco estrategias para la formación y el cambio de actitudes.

III. 19 Conozco a los autores más representativos en el campo de los valores

III. 20 Entiendo las diferencias entre técnica, estrategia, recurso y programa
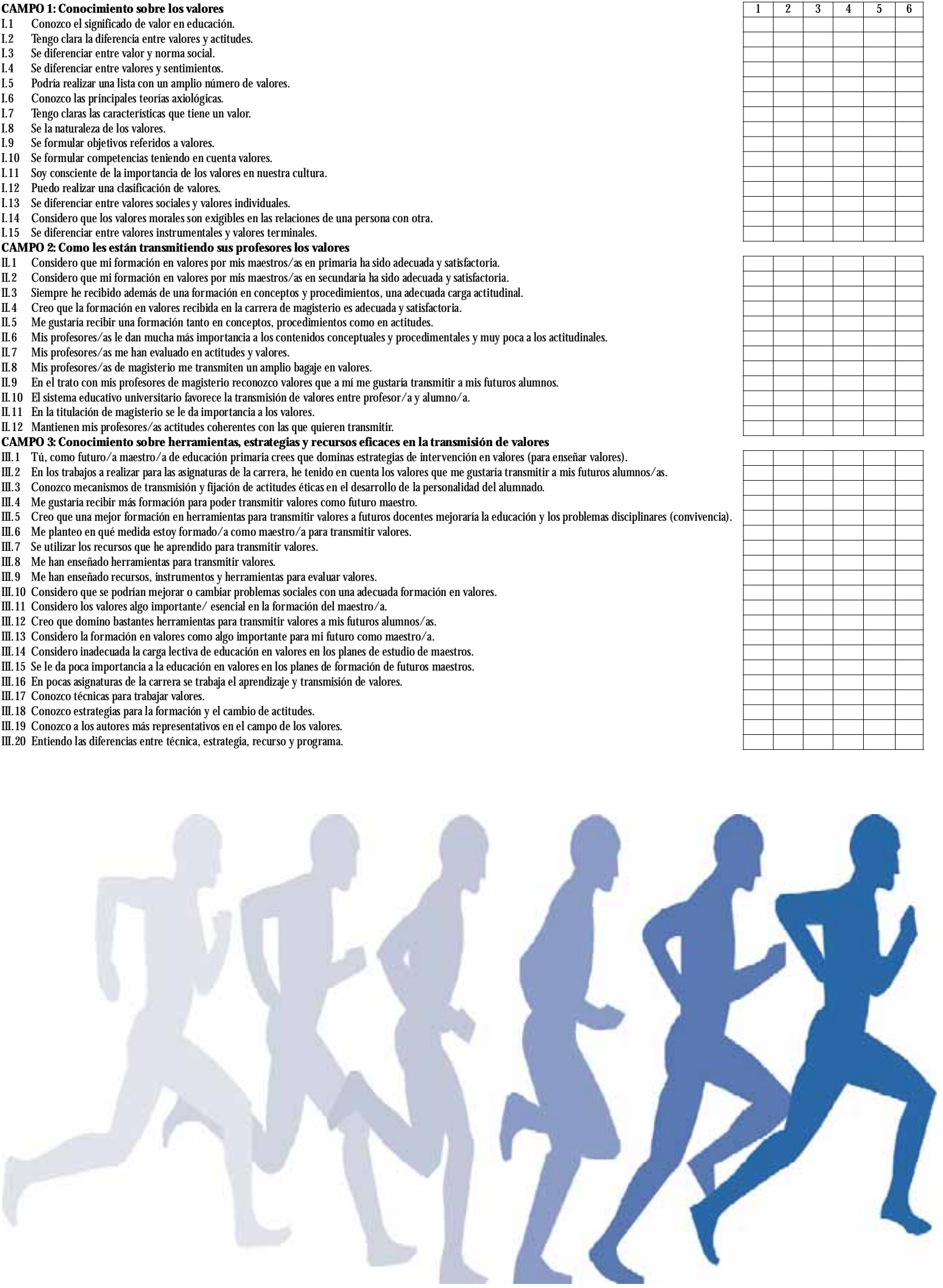